\title{
3D FIB SEM imaging of oil filled chalk: What are the challenges?
}

\author{
K. N. Dalby ${ }^{1}$, R. P. Harti ${ }^{1}$, H. O. Sørensen ${ }^{1}$ and S. L. S. Stipp ${ }^{1}$ \\ 1. NanoGeoScience Group, Department of Chemistry, Nano-Science Center, University of Copenhagen, \\ 2100, Denmark
}

In Europe, fresh groundwater and oil resources are hosted in chalk, which is a sedimentary rock, dominated by calcite $\left(\mathrm{CaCO}_{3}\right)$ precipitated by marine organisms. The calcite particles have remained small during millions of years of compaction and weathering, creating a complex microstructure with low permeability in spite of often high porosity. Knowledge about pore architecture is required to simulate fluid flow, which can be used to predict how contaminants would behave in groundwater aquifers, or how easily oil could be extracted.

Much of the characterisation of chalk porosity was done before the current explosion in high-resolution techniques, resulting in fluid flow models based on information gathered at the centimeter to micrometer scale. However, it is now possible to examine complex porous systems at nanometer resolution [1] and to use the data to improve predictions of petrophysical parameters [2]. X-ray tomography and ptychographic X-ray tomography are pushing resolution boundaries but the focused ion beam scanning electron microscope (FIB SEM) provides some of the highest resolution for imaging natural materials in 3D [3]. A disadvantage of FIB SEM however, is that one cannot image liquid phases because of the need for high vacuum in the analysis chamber. One option is to use a cryogenic stage and freeze the liquids [4]; another is to solidify the oil in place using osmium tetroxide [5], which is the method we explored. An advantage of oil solidification is that freezing is not required and the atomic number contrast is enhanced because Os diffuses into the liquid phase during the stabilisation process (Figure 1).

To investigate the feasibility of using the Os fixation of the oil in chalk investigations, we took three fragments from the same Ålborg chalk. We imaged one dry, another partially filled with oil and another that was fully filled. We used a dual beam FIB SEM and collected information as we milled through the sample, to produce 3D images. Figure 1 illustrates that there are several factors to consider. The first is the heterogeneous nature of the chalk. The completely dry and partially filled images show similar areas of chalk where calcite dominates but the completely oil filled area is dominated by pores. This is the common "representative volume" issue, where input from lower resolution methods is necessary. The second factor is the milling and imaging environment. The effect of beam damage on pore architecture is an important aspect. The choice of detector can help reduce complications in the subsequent image processing. The third factor is that the osmium could modify the pore surface composition, thus affecting the wetting behaviour and oil distribution [6] and the interpretation of pore geometry. By taking these three factors into account, we can begin to visualize pore scale fluid displacement processes, down to nanometer scale. [7] 
[1] K N Dalby et al, Microsc. Microanal. (2014) p. 316.

[2] D Müter et al, Applied Physics Letters 105 (2014), p. 043108.

[3] T L Burnett et al., Scientific Reports 4 (2014), p. 4711

[4] G Desbois and J L Urai., EMC Vol 2: Materials Science (2008), p807.

[5] R P. Harti, University of Copenhagen MSc thesis (2015)

[6] J Gosh and G R Tick, Journal of Contaminant Hydrology 155, p. 20

[7] The authors acknowledge funding from $\mathrm{P}^{3}$ Project, funded by Maersk Oil and Gas A/S and the

Danish National Advanced Technology Foundation and by the Danish Council for Independent

Research (via DANSCATT).
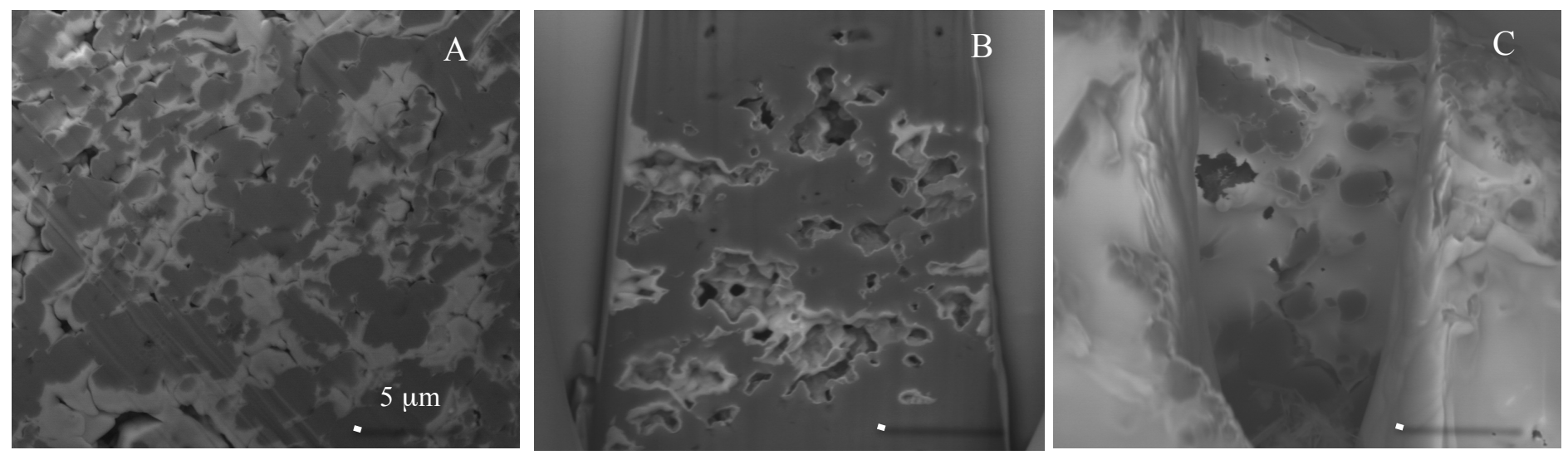

Figure 1. Heterogeneous nature of chalk on the micrometer scale. Back scattered electron images of dry chalk (A), pores partially filled with oil (B) and completely filled (C). Scale is 5 micrometers and images are taken at the same beam settings $(10 \mathrm{kV}$ and $8 \mathrm{nA})$. In all three images, air filled pores are black and calcite is dark grey. EDXS mapping of these sections shows that in the dry sample (A), the lighter contrast material is redeposited calcite rich in Ga. In the partially filled sample (B), the lighter contrast comes from the Os used to stabilise the oil. In the completely filled sample (C), the lighter contrast is provided by a thick Os film that smears with electron beam and ion beam interference. 